\title{
One-year timeline kinetics of cytokine-mediated cellular immunity in dogs vaccinated against visceral leishmaniasis
}

\author{
Christiane Costa-Pereira', Marcela L Moreira', Rodrigo P Soares ${ }^{1}$, Bruno H Marteleto ${ }^{1}$, Vitor M Ribeiro³, \\ Michelle H França-Dias ${ }^{1}$, Ludmila M Cardoso ${ }^{1}$, Kelvinson F Viana ${ }^{2}$, Rodolfo C Giunchetti ${ }^{2}$, Olindo A Martins-Filho ${ }^{1}$ \\ and Márcio S S Araújo ${ }^{1 *}$
}

\begin{abstract}
Background: The main control strategy for visceral leishmaniasis in Brazil has been based on the elimination of seropositive dogs, although this is not widely accepted. In this context, the use of a long-lasting protective vaccine against canine visceral leishmaniasis (CVL) has been highly expected. The aim of this work was to determine the timeline kinetics of the cytokine microenvironment derived from circulating leukocytes as supportive immunological biomarkers triggered by Leishmune ${ }^{\circledast}$ vaccine. Cross-sectional kinetic analysis of cellular immunity cytokines was carried out at three times (1, 6 and 12 months) after primovaccination with Leishmune ${ }^{\oplus}$. In vitro short-term whole blood cultures were stimulated with Leishmania infantum soluble antigen (SLAg). The secreted cytokine signatures and their major sources were determined.

Results: At six months after vaccination, Leishmune ${ }^{\circledast}$ induced an increase in IL-8, IFN- $\gamma$, IL-17a and TNF-a levels and a decrease in IL-10. Cytokine signature analysis revealed a shift in the microenvironment towards a proinflammatory profile mediated by IL-8 and IFN- $\gamma$. Both, CD4 ${ }^{+}\left(\uparrow T N F-a^{+}\right.$and $\left.\uparrow I F N-\gamma^{+}\right)$and CD8 ${ }^{+}(\uparrow I L-17 a$ and $\downarrow \| L-4)$ T-cells contributed to the acquired immune responses observed after stimulation with SLAg.

Conclusions: The changes observed in the cytokine profile suggested that Leishmune ${ }^{\circledR}$ was able to induce an effective response at six months after primovaccination. After one year, it returned to baseline suggesting the need of additional boosting.
\end{abstract}

Keywords: Canine visceral leishmaniasis, Vaccine, Leishmune ${ }^{\circledast}$, Cytokines

\section{Background}

Visceral leishmaniasis (VL), caused by Leishmania infantum (syn. Leishmania chagasi), is the most severe and fatal form of leishmaniasis. Canine visceral leishmaniasis $(\mathrm{CVL})$ is a serious public health problem in the Americas, Mediterranean region, Africa, Asia and Europe [1]. Dogs are very susceptible to infection and the most important domestic hosts [2,3]. Euthanasia of seropositive dogs is recommended, but it did not effectively decrease the number of canine and human cases in Brazil [4]. Although chemotherapy may reduce or eliminate clinical signs in

\footnotetext{
*Correspondence: sobreira@cpqrr.fiocruz.br

'Laboratório de Biomarcadores de Diagnóstico e Monitoração, Centro de Pesquisas René Rachou/FIOCRUZ - MG, Av. Augusto de Lima, 1715,

30190-002 Belo Horizonte, MG, Brazil

Full list of author information is available at the end of the article
}

sick dogs, parasitological cure is not achieved [5]. In fact, a large proportion of infected dogs may become subclinically infected after chemotherapy, but some of them can still transmit the parasite to the sand fly vector. The use of topical insecticides and impregnated collars, as well as vaccines, is difficult to implement in a nationwide control program [6]. In the absence of other successful strategies, vaccine development against CVL has been quoted as one more promising control measure [7]. There is a clear consensus that CVL immunoprofilaxis may also have a positive impact on the human leishmaniasis transmission. However, although some available vaccines decrease infectiousness of the disease for dogs, they still allowed sand fly infection [8]. 
The course of L. infantum infection in dogs depends on the host immune response, persistence and multiplication of the parasite. The innate and adaptive immunity components are engaged in a wide range of complex interactions. The initial steps in the innate immune compartment are important for a successful development of an acquired immune response $[9,10]$. Cytokines play a decisive role during L. infantum infection [11]. Cellular immune responses mediated by pro-inflammatory cytokines (IFN- $\gamma$ and TNF- $\alpha$, were predominant in subclinically infected dogs, suggesting their putative role for protection against the disease. On the other hand, regulatory cytokines (IL-4 and IL-10) seemed to be associated with disease progression and severity [12]. However, there is a consensus that a pro-inflammatory cellular immunity plays a relevant role in the protective events during CVL [12-14].

Many efforts have been made by several groups in order to develop a vaccine against CVL [15-17]. In Brazil, the vaccine Leishmune ${ }^{\circledast}$ (Pfizer-Zoetis) was used for many years. Nowadays, only one commercially available vaccine is in use (Leish-Tec) (Hertape Calier). Leishmune ${ }^{\circ}$ is composed of a glycoproteic complex, fucose-mannose ligand (FML), and Leish-Tec consists of the A2 recombinant protein in saponin. Field studies in Brazil endemic areas demonstrated that Leishmune ${ }^{\odot}$ exhibited 76-80\% efficacy [18-20]. Leish-Tec was also a potent immunobiological tool to prevent CVL, inducing high levels of IgG2 and IFN- $\gamma$ with concomitant decrease of IL-10 [21].

Here, the one-year timeline kinetics of the proinflammatory and regulatory cytokines was evaluated in Leishmune $^{\oplus}$ vaccinated dogs. The cytokine profile produced by circulating leukocytes after short-term in vitro stimulation with LSAg enabled the establishment of supportive immunological biomarkers after primovaccination.

\section{Methods}

\section{Ethics statement}

The study protocol was approved by the Ethical Committee for the Use of Experimental Animals (CEUA) of the Fundação Oswaldo Cruz (FIOCRUZ - PROTOCOL No. P-71/11-3).

\section{Dogs}

In this work, 40 dogs of different breeds (18 males and 22 females), with ages ranging from 8 months to 8 years, were selected in veterinary clinics located in Belo Horizonte, Minas Gerais, Brazil and Informed written consent was obtained from owners of all dogs. All dogs were evaluated during the course of the experiments by their respective Veterinary Doctors. Only healthy dogs from the same area with negative serology (ELISA and Indirect Immunofluorescence - IIF) for CVL and regular anti-helmintic treatment were included in the study. Leishmune ${ }^{\circledast}$ primovaccination was confirmed by immunization cards or in the clinical record files at the veterinary clinics. This is a cross-sectional study structured in three groups of 10 animals each, categorized according time after Leishmune ${ }^{\circ}$ primovaccination (T1, T6 and T12 stand for one, six and twelve months post-vaccination, respectively). All dogs had received subcutaneously the complete immunization protocol proposed by the Leishmune manufacturer (Pfizer-Zoetis, Campinas, and São Paulo, Brazil). This consisted of three shots with 21 days interval. A group of ten seronegative non-vaccinated conscripts was included as control (T0) to evaluate the pro and antiinflammatory basal levels of cytokines.

\section{Leishmania infantum soluble antigen (SLAg)}

Leishmania infantum promastigote forms (MHOM/BR/ 1970/BH46) were grown in liver infusion tryptose medium (LIT), supplemented with $10 \%$ of fetal bovine serum at $24^{\circ} \mathrm{C}$. SLAg production used stationary-phase parasites (7 days of growth) as described [22]. Final protein concentration was adjusted to $1 \mathrm{mg} / \mathrm{mL}$. Aliquots were stored at $-70^{\circ} \mathrm{C}$ prior to short-term in vitro stimulation.

\section{Short-term whole blood culture in vitro}

Five $\mathrm{mL}$ of whole peripheral blood were collected from each dog in heparinized vacuum tubes. In vitro shortterm cultures were performed as described by Silva et al. [23] and whole blood leukocyte counts were determined by automated hematology analyzer (Advia60, Bayer Diagnostics, Tarrytown, NY, USA). Aliquots of $500 \mu \mathrm{L}$ of heparinized peripheral blood (containing $5-7 \times 10^{6}$ whole blood leukocytes) were transferred to lithium heparin Plasma Separator Tubes (Vacutainer ${ }^{\bullet}$ PST tubes, BD, Franklin Lakes, NJ, USA) and incubated for 48 hours at $37^{\circ} \mathrm{C}, 5 \% \mathrm{CO}_{2}$, in the presence of $475 \mu \mathrm{L}$ of RPMI 1640 medium (GIBCO, Grand Island, NY). One tube, referred as Ag-Stimulated Culture (SLAg), received $25 \mu \mathrm{L}$ of $L$. infantum soluble antigen (SLAg at $1 \mathrm{mg} / \mathrm{mL}$, final concentration $25 \mu \mathrm{g} / \mathrm{mL}$ ). A second tube, Control Culture (CC), received $25 \mu \mathrm{L}$ of RPMI. Finally, as internal positive control, was incubated with phorbol myristate acetate (PMA; $25 \mathrm{ng} / \mathrm{mL}$ ) and ionomycin $(1 \mu \mathrm{g} / \mathrm{mL})$ in RPMI. The cultures were incubated at $5 \% \mathrm{CO}_{2}, 37^{\circ} \mathrm{C}$ for 48 hours.

\section{Analysis of peripheral leukocyte secreted cytokines by enzyme-linked immune sorbent assay (ELISA)}

Following short-term whole blood culture in vitro, the tubes were centrifuged $1,400 \mathrm{~g}$ for 10 minutes, and the supernatant stored at $-80^{\circ} \mathrm{C}$ in $100 \mu \mathrm{L}$ aliquots for soluble cytokines quantification by ELISA.

Soluble cytokine levels were determined by DuoSet enzyme-linked immunosorbent assay (ELISA) to quantify IL-8 (anti-canine IL-8, catalog number: DY1608); TNF- $\alpha$ 
(anti-canine TNF- $\alpha$ /TNFSF1A immunoassay; catalog number: DY1507); IFN- $\gamma$ (anti-canine IFN- $\gamma$, catalog number: DY781B) and IL-10 (anti-canine IL-10, catalog number: DY735). Home-standardized ELISA was carried out to quantify IL-4, using monoclonal anti-canine IL-4 antibody (catalog number: MAB7541) as capture antibody; recombinant canine IL-4 (catalog number: 754CL) for obtaining the standard curve and biotinylated anti-canine IL-4 antibody (catalog number: BAF754), avidine peroxydase (R\&D Systems, DY998), and substrate solution (1:1 mixture of $\mathrm{H}_{2} \mathrm{O}_{2}$ and tetramethyl-benzidine, product code 50-76-4, lot. no. RB49).

Briefly, the ELISA assays were carried out by adding $25 \mu \mathrm{L}$ of PBS-diluted monoclonal anti-cytokine to 96 well plates $\left(\mathrm{COSTA}^{\circ}\right.$, Washington, DC, USA), followed by overnight incubation at room temperature. After four wash steps with PBS-Tween 20 buffer, blocking procedures were carry out for $1 \mathrm{~h}$ with $0.1 \%$ of bovine serum albumin (BSA) and $0.05 \%$ sodium azide in PBS. Following four wash steps with PBS-Tween 20 buffer, $25 \mu \mathrm{L}$ of culture supernatant were added to specific wells. Alongside, $25 \mu \mathrm{L}$ of serial diluted recombinant cytokine were used to establish the standard curve. The plates were incubated for $2 \mathrm{~h}$ at room temperature, washed twice with PBS-Tween 20 and $25 \mu \mathrm{L}$ of biotinylated anti-cytokine antibody were added to each well. After two wash steps with PBS-Tween 20, $25 \mu \mathrm{L}$ of avidine peroxydase diluted in PBS $-0.1 \%$ BSA were added to each well and incubated for $30 \mathrm{~min}$ at room temperature. After washing steps with PBS, $25 \mu \mathrm{L}$ of substrate solution $\left(\mathrm{H}_{2} \mathrm{O}_{2}\right.$ and tetramethylbenzidine, 1:1) were added and after 10 minutes $25 \mu \mathrm{L}$ of stopping solution $\left(\mathrm{H}_{2} \mathrm{SO}_{4}, 1 \mathrm{M}\right)$ used as a final reagent. The optical density was determined by automatic Absorbance Microplate Reader (Biotek, EL800, Winosski, VT) at a wavelength of $450 \mathrm{~nm}$. Minimum sensitivity of ELISA were IL-8 $(15.6 \mathrm{pg} / \mathrm{mL}), \mathrm{TNF}-\alpha 15.6 \mathrm{pg} / \mathrm{mL})$, IFN- $\gamma$ $(31.2 \mathrm{pg} / \mathrm{mL})$ and $\mathrm{IL}-10(31.2 \mathrm{pg} / \mathrm{mL})$ as provided by the manufacturer.

\section{Immunophenotypic and intracytoplasmic cytokine staining}

After short-term whole blood culture in vitro, $10 \mu \mathrm{L}$ of Brefeldin A (BFA; Sigma Chemical Company, St. Louis, $\mathrm{MO})$ at $1 \mathrm{mg} / \mathrm{mL}$ was added to each tube and cultures were submitted to an additional incubation for four hours at $5 \% \mathrm{CO}_{2}, 37^{\circ} \mathrm{C}$. Then, $200 \mu \mathrm{L}$ of ethylene diamine tetra acetic acid - EDTA (Sigma, St Louis, USA) were added to each culture tube ( $2 \mathrm{mM}$ final concentration). After $10 \mathrm{mi}-$ nutes of incubation at room temperature, cultured whole blood samples were washed with PBS supplemented with $0.5 \%$ bovine serum albumin and $0.1 \%$ sodium azide (Sigma, St. Louis, USA). The cells were resuspended in $500 \mu \mathrm{L}$ of PBS- $0.5 \%$ BSA. Aliquots of $100 \mu \mathrm{L}$ were stained with anti-cell surface monoclonal antibodies (anti-CD4FITC and anti-CD8-A647, Table 1) for 30 minutes at room temperature. After membrane staining, erythrocyte lysis, and leukocytes fixation, the cell suspension was permeabilized with PBS-0.05\% BSA buffer supplemented with $0.5 \%$ saponin. Aliquots of $50 \mu \mathrm{L}$ were incubated in the presence of phycoerythrin fluorochrome (PE)-labeled anti-cytokines mAbs (IL-17a, TNF- $\alpha$, IFN- $\gamma$, TGF- $\beta$ and IL-4 - Table 1), for 30 minutes at room temperature, in the dark. After intracytoplasmic cytokine staining, the leucocytes were washed with PBS-0.05\% BSA and fixed in FACS fixing solution (10 g/L paraformaldehyde, $10.2 \mathrm{~g} / \mathrm{L}$ sodium cacodylate, and $6.63 \mathrm{~g} / \mathrm{L}$ sodium chloride, $\mathrm{pH}$ 7.2). Flow cytometric measurements were performed on a FACScalibur instrument (Becton Dickson - BD, USA) interfaced to an apple G3 FACStation. The Cell-Quest ${ }^{\text {tm }}$ software package provided by the manufacturer (Franklin Lakes, NJ, USA) was used for data acquisition and analysis. A total of 30,000 events were acquired for each preparation. The frequency of $\mathrm{CD} 4^{+}$and $\mathrm{CD} 8^{+} \mathrm{T}$-cells expressing intracytoplasmic cytokines (IL-17a, TNF- $\alpha$, IFN- $\gamma$, TGF- $\beta$ and IL-4) was determined following the conventional strategy analysis. This analysis consisted of selecting the population of interest, based in morphometric aspects, through punctual distribution of size (forward scatter- FSC) and granularity (side scatter - SSC) graphs. After the selection of the interest region R1 containing FSC ${ }^{\mathrm{Low}} \mathrm{SSC}^{\mathrm{Low}}$ phenotype cells, graphs of density plot distribution of CD4/FL1 or CD8/FL4 versus IL-17a/FL2, TNF- $\alpha / F L 2$, IFN- $\gamma / F L 2$, TGF- $\beta /$ FL2 or IL-4/FL2 were made to determine the percentage of IL- $17 \mathrm{a}^{+}$, TNF- $\alpha^{+}$, IFN- $\gamma^{+}$, TGF- $\beta^{+}$and IL- $4^{+}$ cells inside the previously selected lymphocytes (Figure 1). Final data was expressed as cytokine indexes, calculated by dividing the percentage of cytokine positive cells observed in the SLAg-stimulated culture by the one observed in paired control unstimulated culture (SLAg/CC ratio).

\section{Comparative data analysis \\ Conventional statistical analysis}

The statistical analysis was performed using the software GraphPad Prism 5.03. For the data presenting a parametric distribution, for example, intracytoplasmatic cytokines, variance analysis (ANOVA) was used, followed by Tukey test. For the non-parametric distribution, for example, secreted cytokines in the supernatant, it was performed the Kruskal-Wallis test, followed by Dunns test. Differences were considered significant at $\mathrm{p} \leq 0.05$.

\section{Comparative analysis of cytokines signatures secreted by peripheral leucocytes}

Cytokine signatures were compared to characterize the pattern of secreted cytokines from each animal vaccinated as suggested [23,24]. Briefly, the "global median" value for each secreted cytokine was calculated by taking the whole universe of data $(\mathrm{T} 0+\mathrm{T} 1+\mathrm{T} 6+\mathrm{T} 12)$. The "global median" was used as the cut off to tag each dog 
Table 1 Monoclonal antibodies used for immunophenotyping assays intracytoplasmic detection of cytokines in vitro

\begin{tabular}{lllll}
\hline Marker & Host & Clone & Fluorochrome & Manufacturer \\
\hline Canine Anti-CD4 & Rat & YKIX302.9 & FITC & Serotec \\
Canine Anti-CD8 & Rat & YCATE55.9 & A647 & Serotec \\
Human Anti-IL17-a & mouse & 64 DEC17 & R-PE & BD Pharmingen \\
Human Anti-TNF-a & mouse & MAb11 & R-PE & BD Pharmingen \\
Bovine Anti-IFN- $\gamma$ & mouse & CC302 & R-PE & Serotec \\
Human Anti-TGF- $\beta$ & mouse & TB21 & R-PE & Q Products \\
Bovine Anti-IL 4 & mouse & CC303 & R-PE & Serotec \\
\hline
\end{tabular}

as they display "Low" or "High" levels of secreted cytokines in the culture supernatant as follows: (IL-8 = $2,087 \mathrm{pg} / \mathrm{mL} ;$ TNF- $\alpha=761 \mathrm{pg} / \mathrm{mL} ;$ IFN $-\gamma=929 \mathrm{pg} / \mathrm{mL}$; $\mathrm{IL}-4=57 \mathrm{pg} / \mathrm{mL}$ and IL-10 $=135 \mathrm{pg} / \mathrm{mL}$ ) (Figure 2A). Following the assembling of dogs categorized as "Low" or "High" cytokines producers, the frequency was calculated using gray-scale diagrams for each timeline (T0, T1, T6 and T12) (Figure 2B). The ascendant frequency of high cytokine for each timeline was assessed to generate the T0, T1, T6 and T12 cytokine signatures (bar charts) (Figure 2C). Relevant frequencies of "high cytokine producers" were considered when the

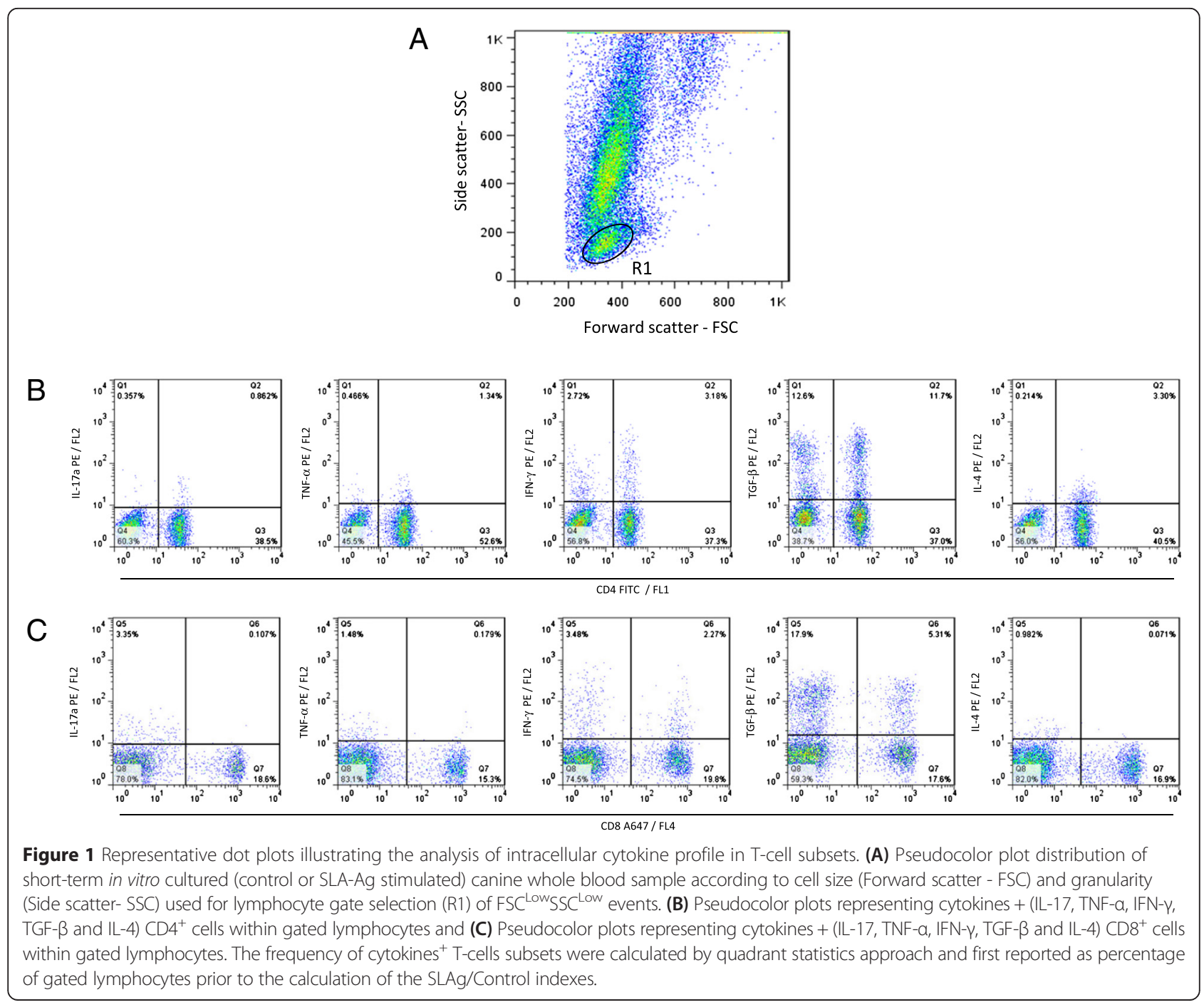


A

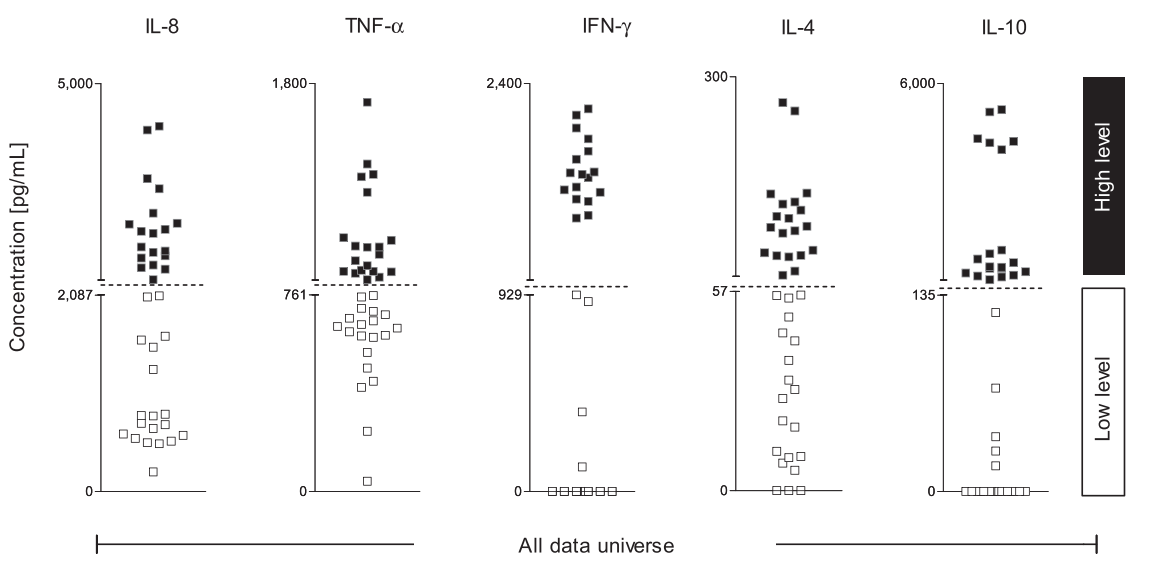

B
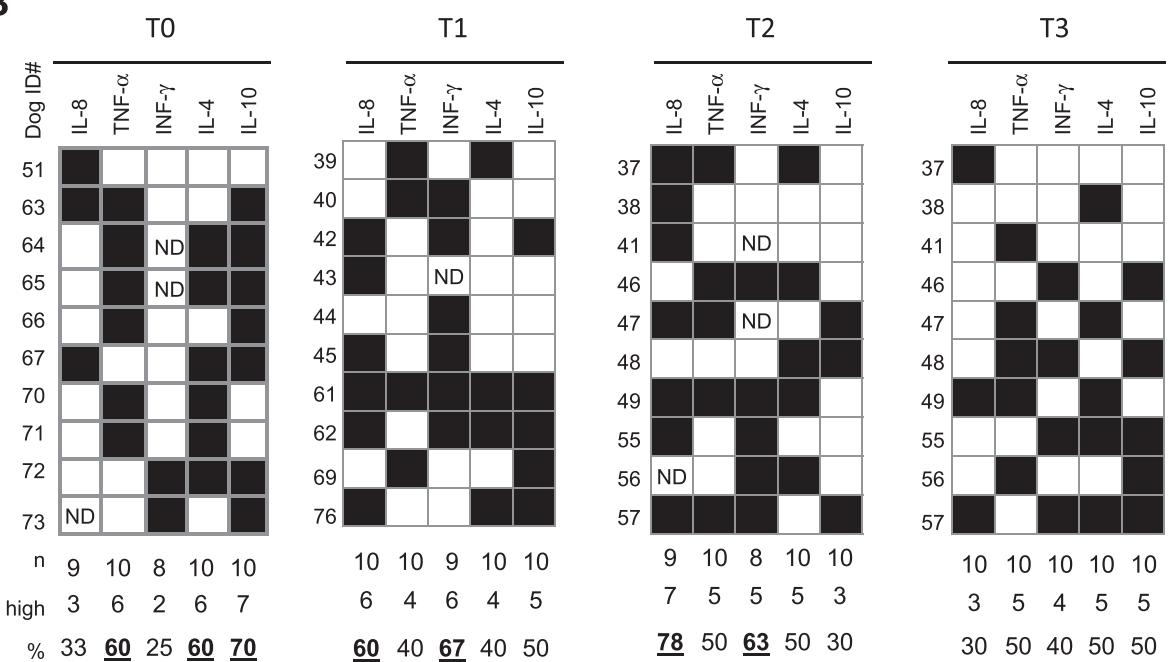

C

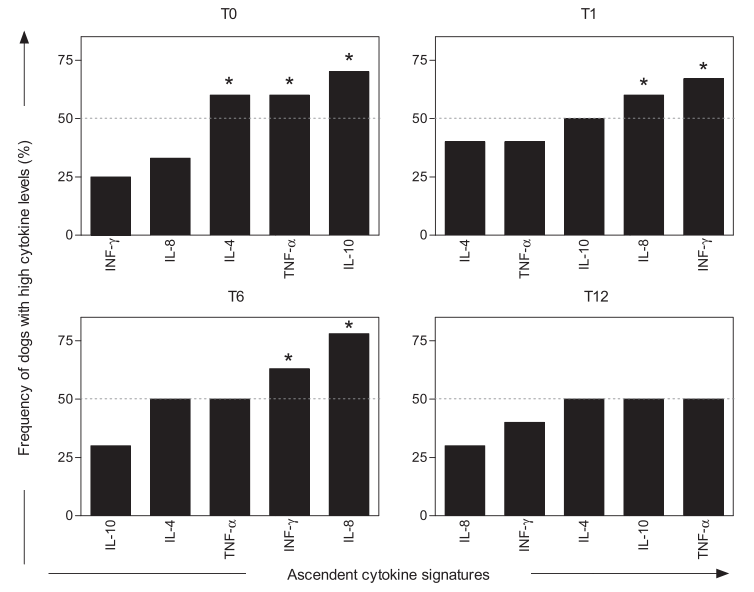

D

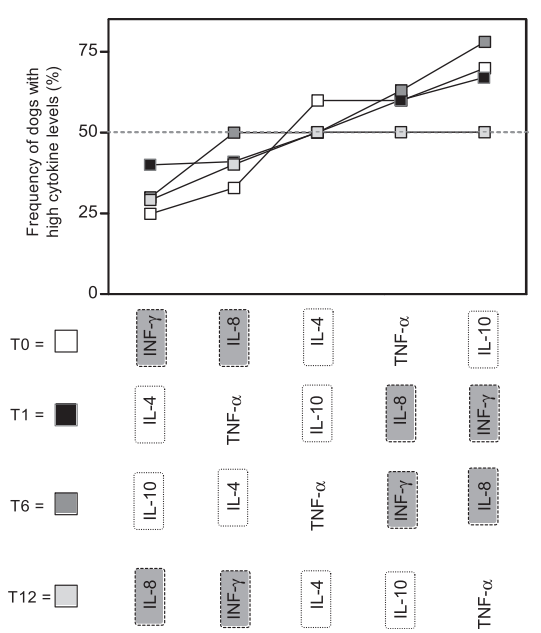

Figure $\mathbf{2}$ (See legend on next page.) 
(See figure on previous page.)

Figure 2 Signature analysis of secreted cytokine by peripheral blood leukocytes afterin vitrostimulation withLeishmania infantum soluble antigens (SLAg). (A) Establishment of the global median cut-off edges for each cytokine (IL-8, TNF-a, IFN- - , IL-4 and IL-10) used to segregate dogs as they present "Low" ( $\square$ ) or "High" ( cytokine levels. (B) Gray-scale diagrams used to compile the frequency (\%) of high cytokine producers.

(C) Ascendant cytokine signatures were assembled for each time after vaccination (T0, T1, T6 and T12). The frequencies of high producers were considered relevant $\left(^{*}\right)$ when the percentage was confined over the $50^{\text {th }}$ percentile (doted lines). (D) Comparative analysis of cytokine signatures were used to identify relevant differences amongst Leishmune ${ }^{\oplus}$ vaccinated dogs at one $(T 1=$ black rectangle), six ( $T 6=$ dark grey rectangle) and twelve (T12 = light grey rectangle) months post-vaccination compared to unvaccinated dogs (T0 = white rectangle). Gray scale rectangles were used to highlight the shift in the overall profile of pro-inflammatory and regulatory cytokines on each time after immunization. Relevant differences (shift of frequencies across the $50^{\text {th }}$ percentile cut-off edge) were highlighted by gray background.

percentage was above the $50^{\text {th }}$ percentile. In a final step, the cytokine signatures were converted from the bar chart to line curves for overlaid comparative analysis amongst each timeline (T0, T1, T6 and T12). Relevant differences (shift of frequencies across the $50^{\text {th }}$ percentile cut-off edge) were highlighted by gray background (Figure 2D).

\section{Results}

Timeline kinetics of cytokines secreted by peripheral blood leukocytes after in vitro stimulation

After stimulation with SLAg, a peak of leukocyte-secreted pro-inflammatory cytokines (IL-8 an IFN- $\gamma$ ) was observed at T6. This was concomitant with a decrease of IL-10 compared to non-immunized dogs. Moreover, it was

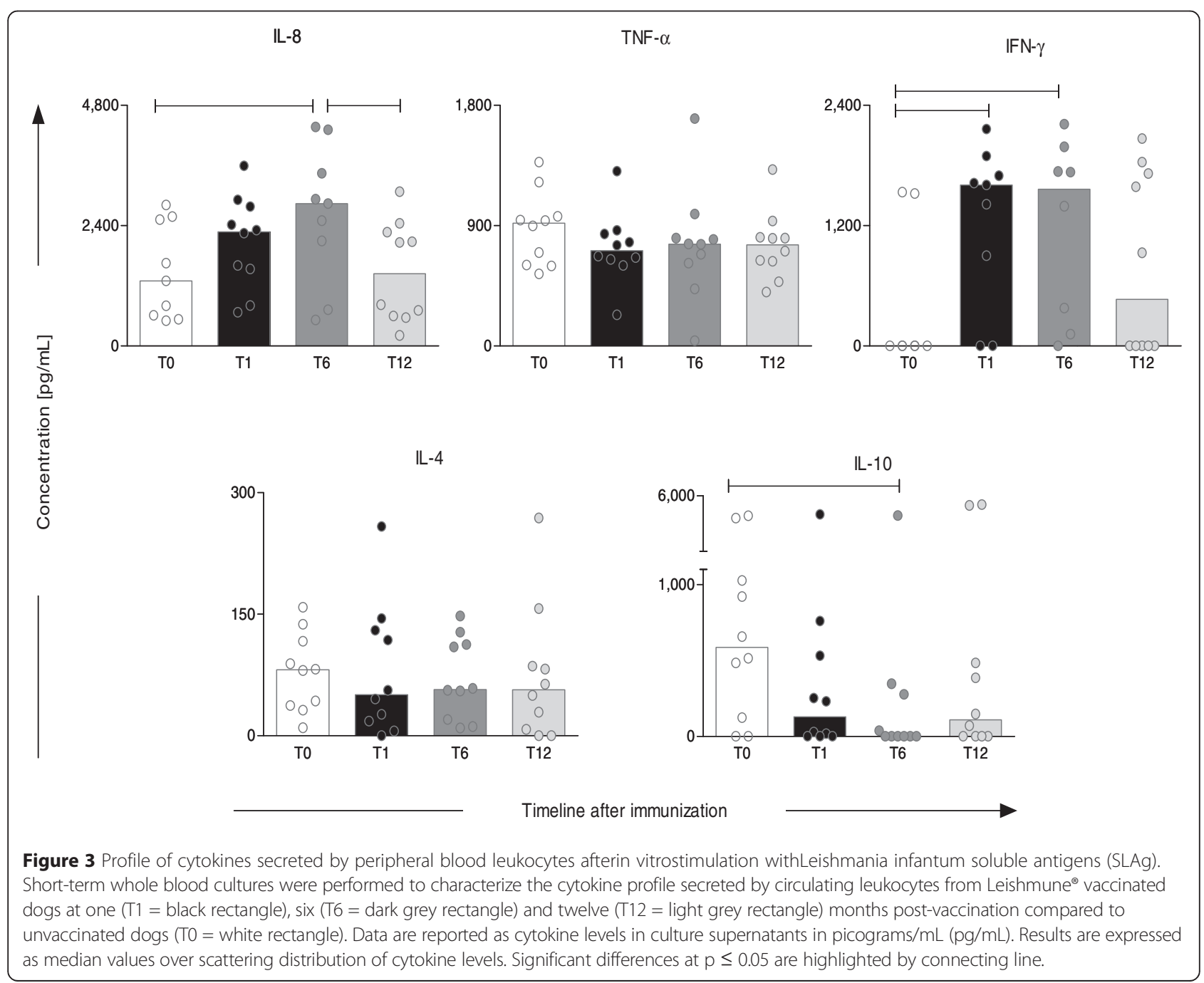


noticed that IL-8 levels return to baseline at T12. More importantly, IFN- $\gamma$, a relevant immunological biomarker, was significantly increased at $\mathrm{T} 1$, re-enforcing the ability of Leishmune ${ }^{\circ}$ to trigger a protection pattern in primovaccinees. As expected, there is some basal production of cytokines by the dogs at T0 (Figure 3).

\section{Cytokine signature analysis further demonstrated that} Leishmune ${ }^{\circledR}$ vaccination shifted the immune response towards a pro-inflammatory profile

After cytokine signature analysis (Figure 2), the frequency of high producers was determined for each experimental group of animals. This qualitative data re-emphasized the supportive immune protection biomarkers observed after Leishmune $^{\ominus}$ vaccination. A shift towards pro-inflammatory cytokines (IFN- $\gamma$ and IL-8) was observed from T0 to T1 and sustained at T6. Concomitant decrease of regulatory cytokines (IL-4 and IL-10) was also observed at T1 and T6 compared to T0. Moreover, it was important to notice that at T12, the cytokine signatures returned to baseline profile similar to T0, with enhanced frequency of dogs presenting high levels of IL-10 and IL-4.

\section{Intracytoplasmic cytokine indexes in peripheral blood leukocytes from Leishmune ${ }^{\circledast}$ vaccinated dogs after in vitro stimulation with SLAg}

In order to identify the major cytokine sources induced in peripheral blood leukocytes after in vitro stimulation, each dog cytokine indexes observed for T-cells and Tcell subsets were calculated (Figure 4). A clear peak of IL-17a, TNF- $\alpha$ derived from T-cells at T1 was observed compared to other groups. Moreover, a decreased index of IL-4 was observed at T6 compared to T1 (Figure 4A). Analysis of $\mathrm{CD}^{+}{ }^{+} \mathrm{T}$-cells demonstrated a peak of TNF- $\alpha$

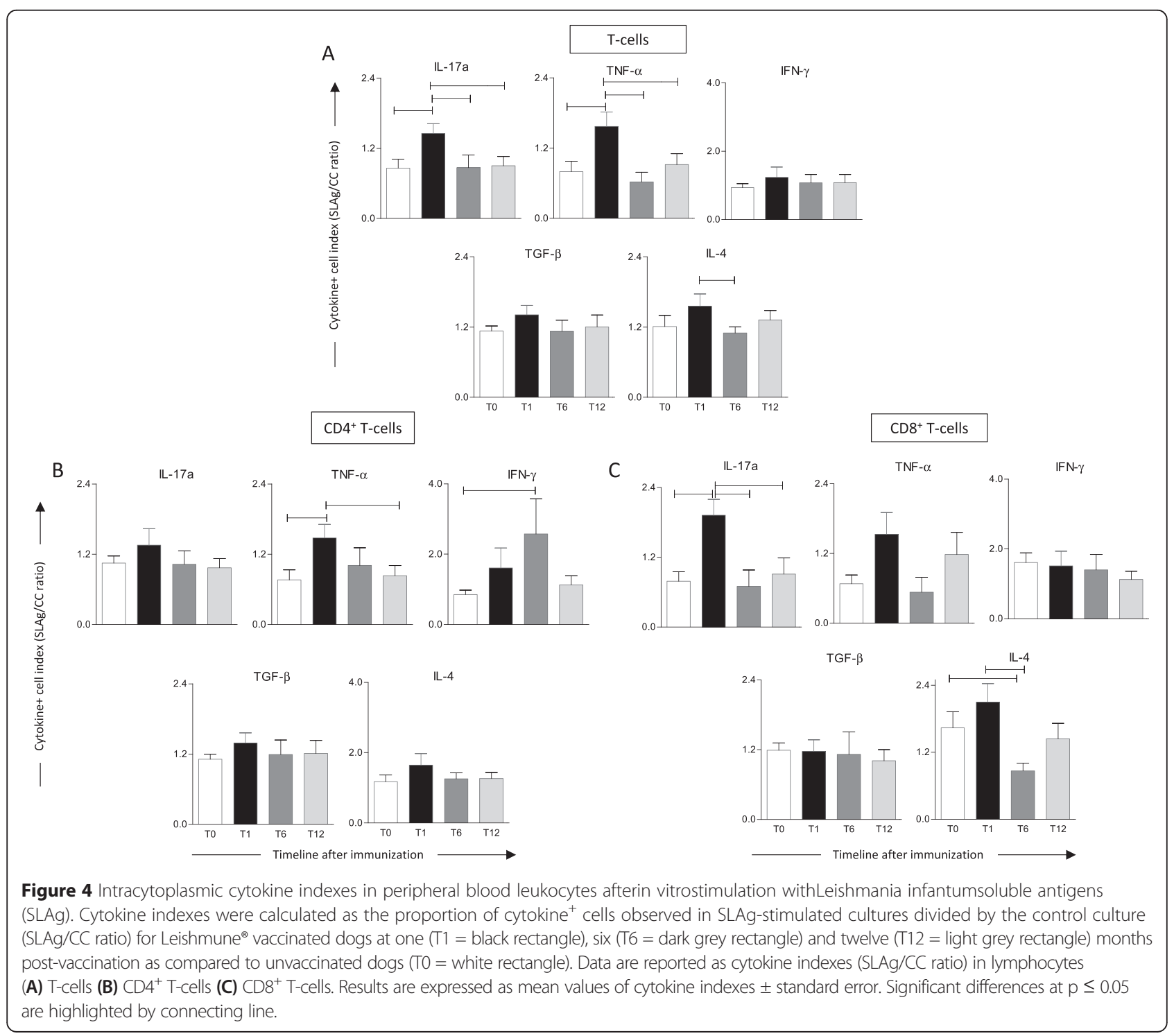


production at $\mathrm{T} 1$ compared to unvaccinated dogs. This cytokine returned to baseline at T12 (Figure 4B). The analysis of IFN- $\gamma$ further demonstrated a peak at T6 (Figure 4B). Analysis of $\mathrm{CD}^{+} \mathrm{T}$-cells revealed a clear peak of IL-17a at T1 compared to other groups and a decrease in the IL-4 index at T6 compared to T1 and T0 (Figure 4C). Together, these findings showed that $\mathrm{T}$ cells, both $\mathrm{CD}^{+}$and $\mathrm{CD} 8^{+}$subsets are relevant sources of pro-inflammatory cytokines demonstrating the ability of Leishmune ${ }^{\bullet}$ to trigger a protective immunological pattern in primovaccinees.

\section{Discussion}

Previous reports have demonstrated the long lasting protection against CVL using Leishmune ${ }^{\circ}$ in endemic areas [19]. This investigation evaluated the kinetics of the proinflammatory and regulatory cellular immune response in Leishmune ${ }^{\bullet}$ vaccinated dogs in a period of one year.

The immunocompetent host is able activate innate and acquired inflammatory mechanisms responsible for disease protection. In dogs, the cellular immune responses mediated by phagocytes and T-cells, primarily involving pro-inflammatory mediators (TNF- $\alpha$, IL- 6, IL-12 and IFN- $\gamma$ ) and toxic oxygen intermediates are relevant in this context. On the other hand, an impaired cellular response is consistent with susceptibility [14,25]. Here, we have evaluated by a cross-sectional investigation, the cytokine profile triggered by Leishmune ${ }^{\circ}$. The timeline kinetics of major pro-inflammatory and regulatory cytokines was determined over a one year period. The data observed would provide supportive insights to the animal health authorities regarding the vaccination guidelines currently recommended for the Leishmune ${ }^{\circ}$ vaccine.

Our data demonstrated that a clear increase in IL-8 and IFN- $\gamma$ in the group of Leishmune $e^{\circ}$ vaccinated dogs at 6 months post-vaccination. In fact, IFN- $\gamma$ increased in the first month after vaccination compared to non-vaccinated dogs. However, the increase of IL-8 and IFN- $\gamma$ did not last after 12 months, showing similar levels to non-vaccinated dogs. On the other hand, a decrease in IL-10 was observed at six months after vaccination, returning to similar levels of the non-vaccinated dogs at T12.IL-8 is essential for neutrophil activity during the early events of the immune response against Leishmania [26]. In our previous studies we have demonstrated that Leishmune ${ }^{\circ}$ vaccination induced an increase in IFN- $\gamma$ production by $\mathrm{CD} 4^{+} \mathrm{T}$-cells $[22,27]$. The increase of those two cytokines in vaccinated dogs is consistent with the ability of Leishmune ${ }^{\circ}$ to trigger an immune response. In this context, they could be classified as immuneprotection biomarkers, since they returned to baseline at T12. The regulatory cytokine IL-10 is known to modulate the pro-inflammatory immune response, inhibiting macrophages and promoting intracellular infection. Its low levels at T6 could support a pro-inflammatory immune profile, enabling parasite elimination [28].

In this study, one innovative tool $[23,24]$ was also used to evaluate the cytokine profiles triggered by Leishmune ${ }^{\circ}$. Cytokine signature analysis revealed a shift in the cytokine milieu after SLAg stimulation. Similar to our previous observations, a shift towards a proinflammatory profile, especially mediated by IL-8 and IFN- $\gamma$ was observed at T1 and T6. Also, signature analysis showed a clear decreased in those cytokines at T12 with a simultaneous increase in the frequency of regulatory cytokines, at similar levels to non-vaccinated dogs. This qualitative approach also re-enforced the use of some of those leukocyte secreted cytokines as supportive immuneprotection biomarkers. Those may be useful to follow vaccination protocols after SLAg stimulation.

Leishmania elimination by phagocytes is a crucial step for infection control and several reports have demonstrated that their effective and persistent activation are controlled by cytokines derived from the adaptive immunity $[29,30]$. In this context, it is likely to hypothetize that the post vaccination memory mediated by adaptive immunity cells may be important for an effective activity of the innate immune mechanisms. It has been proposed that $\mathrm{CD} 4^{+} \mathrm{T}$-cells play a decisive role in this process through the production of cytokines and effectors mechanisms [31]. Consistent with those observations, the analysis of the intracytoplasmic cytokine profiles in T-cell subsets supported these findings. Leishmune $e^{\oplus}$ vaccinated dogs showed increased indexes of IL-17a and TNF- $\alpha$ early (one month) after primovaccination. This is similar to previous studies showing that those cytokines were related to a CVL resistance phenotype $[32,33]$. The analysis of T-cell subpopulations further demonstrated that $\mathrm{CD} 4^{+} \mathrm{T}$-cells were relevant sources of TNF- $\alpha$ and IFN- $\gamma$. At the same time, $\mathrm{CD} 8^{+} \mathrm{T}$-cells were an important source of IL-17a and responsible for IL4decrease. The latter is associated with a regulatory response modulating the pro-inflammatory profile, inhibiting macrophage activity and promoting intracellular infection [28]. Our results also showed an important increase of IFN- $\gamma$ in $\mathrm{CD}^{+}{ }^{+} \mathrm{T}$-cells after in vitro stimulation with SLAg. These results were similar to those observed by $L$. infantum infected macrophages from dogs immunized with the vaccine LiESAp (constituted of purified excreted antigen of L. infantum promastigotes plus muramyl dipeptide as adjuvant). Additionally, their co-cultivation with total lymphocytes results in higher levels of IFN- $\gamma[34,35]$. However, our data showed a clear contribution of both $\mathrm{CD} 4^{+}$and $\mathrm{CD}^{+}$lymphocytes for the acquired immune responses after stimulation with SLAg.

\section{Conclusions}

The data observed showed that Leishmune ${ }^{\bullet}$ was able to trigger changes in the immune response profiles in 
vaccinated dogs especially after 6 months. Although it provided relevant information regarding Leishmune ${ }^{\circ}$ potential and immunization time, one of the limitations of this study relies on the genetic variability of the dogs. Although they had different characteristics (sex, age and breed), it was clearly demonstrated that Leishmune ${ }^{\bullet}$ vaccination was able to shift the immune response towards a pro-inflammatory profile. However, this was important to validate the vaccine status in the real context. The important finding that all biomarkers (IL-8, TNF- $\alpha$, IFN- $\gamma$, IL-17a, IL-4 and IL-10) returned to baseline after one year post vaccination re-enforced the need of boosting doses. Also, it suggests their putative use as immune protection biomarkers for vaccines evaluation. The continuity of this and other studies may help to generate information to understand the immune response mechanisms during CVL immunoprophylaxis.

\section{Abbreviations}

BFA: Brefeldin A; CC: Control culture; CVL: Canine visceral leishmaniasis; EDTA: Ethylene diamine tetra acetic acid; ELISA: Enzyme linked immune sorbent assay; IFN-ү: Interferon gama; IgG2: Immunoglobulin G2; IIF: Indirect immunofluorescence; IL-4: Interleukin 4; IL-8: Interleukin 8; IL-10: Interleukin 10; IL-17a: Interleukin 17a; LIT: Infusion tryptose medium; PBS: Phosphate buffered saline; PMA: Phorbol myristate acetate; SLAg: Leishmania infantum soluble antigens; TGF- $\beta$ : Transforming growth factor beta; TNF-a: Tumor necrosis factor-alpha; T0: Group non-vaccinated; T1: Group one month post-vaccination; T6: Group six months post-vaccination; T12: Group twelve months post-vaccination; VL: Visceral leishmaniasis.
\end{abstract}

\section{Competing interests}

The authors declare that they have no competing interests.

\section{Authors' contributions}

CCP, RPS, OAMF and MSSA designed and prepared the manuscript. CCP, MLM, BHM, VMR, MHFD, LMC and KFV performed the experiments. OAMF, RCG and MSSA supervised the study. All authors have read and approved the final manuscript.

\section{Acknowledgements}

The authors would like to thanks the members of the Laboratório de Biomarcadores de Diagnóstico e Monitoração do Centro de Pesquisas René Rachou, FIOCRUZ-MG for providing invaluable technical assistance during this study. The authors thank the program for technological development in tools for health-PDTIS-FIOCRUZ for the use of its facilities. This work was supported by Conselho Nacional de Desenvolvimento Científico e Tecnológico (CNPq), PAPES/FIOCRUZ, Fundação de Amparo à Pesquisa do Estado de Minas Gerais (FAPEMIG), and Centro de Pesquisas René Rachou (CPqRR/FIOCRUZ-MG). OAMF, RPS and RCG are research fellows from (CNPq).

\section{Author details}

'Laboratório de Biomarcadores de Diagnóstico e Monitoração, Centro de Pesquisas René Rachou/FIOCRUZ - MG, Av. Augusto de Lima, 1715, 30190-002 Belo Horizonte, MG, Brazil. '2Laboratório de Biologia das Interações Celulares, Departamento de Morfologia, Universidade Federal de Minas Gerais, Av. Antônio Carlos, 6627, 31270-901 Belo Horizonte, MG, Brazil. ${ }^{3}$ Clínica Veterinária Santo Agostinho, Avenida Amazonas, 2218, 30180-00 Belo Horizonte, MG, Brazil.

Received: 3 December 2014 Accepted: 17 March 2015

Published online: 11 April 2015

\section{References}

1. WHO. Control of the Leishmaniases: report of a meeting of the WHO Expert Committee on the Control of Leishmaniases, Geneva, 22-26 March 2010. World Health Organization. WHO Tech Rep Ser. 2010;949:1-186.
2. Desjeux P. Leishmaniasis: current situation and new perspectives. Comp Immunol Microbiol Infect Dis. 2004;27:305-18.

3. Dantas-Torres F. The role of dogs as reservoirs of Leishmania parasites, with emphasis on Leishmania (Leishmania) infantum and Leishmania (Viannia) braziliensis. Vet Parasitol. 2007;149:139-46.

4. Maia-Elkhoury AN, Alves WA, Sousa-Gomes ML, Sena JM, Luna EA. Visceral leishmaniasis in Brazil: trends and challenges. Cad Saude Publica. 2008:24:2941-7.

5. Baneth G, Shaw SE. Chemotherapy of canine leishmaniosis. Vet Parasitol. 2002:106:315-24.

6. Quinnell RJ, Courtenay O. Transmission, reservoir host and control of zoonotic visceral leishmaniasis. Parasitology. 2009;136:1915-34.

7. Gramiccia M, Gradoni L. The current status of zoonotic leishmaniases and approaches to disease control. Int J Parasitol. 2005;35:1169-80.

8. Bongiorno G, Paparcone R, Foglia Manzillo V, Oliva G, Cuisinier AM, Gradoni L. Vaccination with LiESP/QA-21 (CaniLeish ${ }^{\odot}$ ) reduces the intensity of infection in Phlebotomusperniciosus fed on Leishmania infantum infected dogs a preliminary xenodiagnosis study. Vet Parasitol. 2013;197:691-5.

9. Moreno J, Alvar J. Canine leishmaniasis: epidemiological risk and the experimental model. Trends Parasitol. 2002;18:399-405.

10. Gazzinelli RT, Ropert C, Campos MA. Role of the Toll/interleukin-1 receptor signaling pathway in host resistance and pathogenesis during infection with protozoan parasites. Immunol Rev. 2004;201:9-25.

11. Carrillo E, Moreno J. Cytokine profiles in canine visceral leishmaniasis. Vet Immunol Immunopathol. 2009:128:67-70.

12. Barbiéri CL. Immunology of canine leishmaniasis. Parasite Immunol. 2006;28:329-37.

13. Alvar J. Canine leishmaniasis. Adv Parasitol. 2004;57:1-88.

14. Reis AB, Teixeira-Carvalho A, Giunchetti RC, Guerra LL, Carvalho MG, Mayrink $W$, et al. Phenotypic features of circulating leucocytes as immunological markers for clinical status and bone marrow parasite density in dogs naturally infected by Leishmania chagasi. Clin Exp Immunol. 2006;146:303-11.

15. Lemesre JL, Holzmuller P, Cavaleyra M, Goncalves RB, Hottin G, Papierok G. Protection against experimental visceral leishmaniasis infection in dogs immunized with purified excreted secreted antigens of Leishmania infantum promastigotes. Vaccine. 2005;23:2825-40.

16. Palatnik-de-Sousa CB. Vaccines for leishmaniasis in the fore coming 25 years, Vaccine. 2008:26:1709-24

17. Nagill R, Kaur S. Vaccine candidates for leishmaniasis: a review. Int Immunopharmacol. 2011:11:1464-88.

18. da Silva VO, Borja-Cabrera GP, Correia Pontes NN, de Souza EP, Luz KG, Palatnik M, et al. A phase III trial of efficacy of the FML-vaccine against canine kalaazar in an endemic area of Brazil (Sao Goncalo do Amaranto, RN). Vaccine. 2000;19:1082-92.

19. Borja-Cabrera GP, Correia Pontes NN, da Silva VO, ParaguaideSouza E, Santos WR, Gomes EM, et al. Long lasting protection against canine kala-azar using the FML-QuilA saponin vaccine in an endemic area of Brazil (Sao Goncalo do Amarante, RN). Vaccine. 2002;20:3277-84

20. Borja-Cabrera GP, Santos FN, Bauer FS, Parra LE, Menz I, Morgado AA, et al. Immunogenicity assay of the Leishmune vaccine against canine visceral leishmaniasis in Brazil. Vaccine. 2008:26:4991-7.

21. Fernandes AP, Costa MM, Coelho EA, Michalick MS, de Freitas E, Melo MN, et al. Protective immunity against challenge with Leishmania (Leishmania) chagasi in beagle dogs vaccinated with recombinant A2 protein. Vaccine. 2008;29:5888-95.

22. Araujo MS, de Andrade RA, Sathler-Avelar R, Teixeira-Carvalho A, Andrade $M C$, Vianna $L R$, et al. T-cell-derived cytokines, nitric oxide production by peripheral blood monocytes and seric anti-Leishmania (Leishmania) chagasi lgG subclass patterns following immunization against canine visceral leishmaniasis using Leishvaccine and Leishmune. Vaccine. 2009;27:1008-17.

23. Silva ML, Martins MA, Espírito-Santo LR, Campi-Azevedo AC, Silveira-Lemos D, Ribeiro JG, et al. Characterization of main cytokine sources from the innate and adaptive immune responses following primary 17DD yellow fever vaccination in adults. Vaccine. 2011;29:583-92.

24. Estrela-Lima A, Araújo MSS, Soares RP, Ribeiro LGR, Damasceno KA, Costa AT, et al. Plasma Biomarkers Profile of female dogs with mammary carcinoma and its association with clinical and pathological features. Vet Comp Oncology. 2014. (in press).

25. Chamizo C, Moreno J, Alvar J. Semi-quantitative analysis of cytokine expression in asymptomatic canine leishmaniasis. Vet Immunol Immunopathol. 2005;103:67-75. 
26. Reis AB, Giunchetti RC, Carrillo E, Martins-Filho AO, Moreno J. Immunity to Leishmania and the rational search for vaccines against canine leishmaniasis. Trends Parasitol. 2010;26:341-9.

27. Araujo MS, Andrade RA, Satheler-Avelar R, Magalhaes CP, Carvalho AT, Andrade MC, et al. Immunological changes in canine peripheral blood leukocytes triggered by immunization with first or second generation vaccines against canine visceral leishmaniasis. Vet Immunol Immunophathol. 2011;141:64-75

28. Murray YHW, Lu CM, Mauzes, Freeman NS, Moreira AL, Kaplan G, et al. Interleukin-10 (IL-10) in experimental visceral leishmaniasis and IL-10 receptor blockade as immunotherapy. Infect Immun. 2002;70:6284-93.

29. Vouldoukis I, Drapier JC, Nüssler AK, Tselentis Y, Da Silva OA, Gentilini M, et al. Canine visceral leishmaniasis: successful chemotherapy induces macrophage antileishmanial activity via the L-arginine nitric oxide pathway. Antimicrob Agents Chemother. 1996:40:253-6.

30. Pinelli E, Gebhard D, Mommaas AM, van Hoeij M, Langermans JA, Ruitenberg EJ, et al. Infection of a canine macrophage cell line with leishmania infantum: determination of nitric oxide production and anti-leishmanial activity. Vet Parasitol. 2000;92:181-9.

31. Kemp K, Kemp M, Kharazmi A, Kurtzhals JAL, Theander TG. Leishmaniaspecific T cells expressing interferon-gamma (IFN- - ) and IL-10 upon activation are expanded in individuals cured of visceral leishmaniasis. Clin Exp Immunol. 1999; 116:500-4.

32. Pinelli E, Ellick-Kendrick R, Wagenaar J, Bernadina W, Real G, Ruitenberg J. Cellular and humoral immune responses in dogs experimentally and naturally infected with Leishmania infantum. Infec Immun. 1994:62:229-35.

33. Alves CF, de Amorim IF, Moura EP, Ribeiro RR, Michalick MS, Kalapothakis E, et al. Expression of IFN-gamma, TNF-alpha, IL-10 and TGF-beta in lymph nodes associates with parasite load and clinical form of disease in dogs naturally infected with Leishmania (Leishmania) chagasi. Vet Immunol Immunopathol. 2009;128:349-58.

34. Holzmuller P, Cavaleyra M, Moreaux J, Kovacic R, Vincendeau P, Papierok G, et al. Lymphocytes of dogs immunised with purified excreted-secreted antigens of Leishmania infantum co-incubated with Leishmania infected macrophages produce IFN gamma resulting in nitric oxide-mediated amastigote apoptosis. Vet Immunol Immunopathol. 2005;106:247-57.

35. Lemesre JL, Holzmuller P, Gonçalves RB, Bourdoiseau G, Hugnet C, Cavaleyra $M$, et al. Long-lasting protection against canine visceral leishmaniasis using the LiESAp-MDP vaccine in endemic areas of France: double-blind randomised efficacy field trial. Vaccine. 2007;21:4223-34.

\section{Submit your next manuscript to BioMed Central and take full advantage of:}

- Convenient online submission

- Thorough peer review

- No space constraints or color figure charges

- Immediate publication on acceptance

- Inclusion in PubMed, CAS, Scopus and Google Scholar

- Research which is freely available for redistribution

Submit your manuscript at www.biomedcentral.com/submit 